\title{
Modeling and simulation of an impact test using wavelets, analytical solutions and finite elements
}

\author{
P. Vandergheynst ${ }^{\mathrm{a}, 1}$, J.-P. Antoine ${ }^{\mathrm{a}}$, E. Van Vyve ${ }^{\mathrm{b}}$, A. Goldberg ${ }^{\text {c }}$, I. Doghri ${ }^{\mathrm{b}, *}$ \\ a Université Catholique de Louvain, Institut de Physique Théorique, 2, chemin du Cyclotron, B-1348 Louvain-la-Neuve, Belgium \\ ${ }^{\mathrm{b}}$ Université Catholique de Louvain, Batiment Euler, Center for Systems Engineering and Applied Mechanics (CESAME), 4, avenue G. \\ Lemaitre, B-1348 Louvain-la-Neuve, Belgium \\ ${ }^{\mathrm{c}}$ Solvay S.A., Laboratoire Central, 310, rue de Ransbeek, B-1120 Bruxelles, Belgium
}

Received 7 July 2000

\begin{abstract}
An instrumented falling weight impact test is thoroughly investigated in two cases, that of a rebound and that of a rupture in the sample. First, the force signal is analyzed with four methods: the continuous wavelet transform (with different wavelets: derivatives of a Gaussian and Morlet's), the Gabor transform, the Wigner-Ville transform and the classical Fourier analysis. It is shown how a proper use of the time-frequency methods allows to precisely detect the discontinuities in the signal, the moment of rupture, if any, and the frequencies that are excited at the impact. In a second part, modal and stress analyses are carried out, both by an analytical method and by finite element computations. The results confirm those obtained in the first part and agree with experimental findings. (c) 2001 Elsevier Science Ltd. All rights reserved.
\end{abstract}

Keywords: Impact; Signal analysis; Wavelets; Finite elements

\section{Introduction}

The technique of the instrumented falling weight impact testing (IFWI) really started to develop with the progress of electronic boards (beginning of the 80s) able to record short time events (typical order of magnitude: $10^{-3}$ s) (see Savadori (1985) for a review).

During an IFWI test, a clamped sample (a disk in general) is hit by a striker of mass $M$ at a certain initial velocity $v_{0}$ (see Fig. 1). During this event, three variables are recorded: the force $F$, the time $t$ and the initial velocity $v_{0}$ (the mass $M$ has been measured previously). Then the displacement and energy can be computed, from momentum conservation. This gives some results that can be compared to model values (with some precaution due to the lack of a calibration procedure). This type of event occurs on a very short time

\footnotetext{
${ }^{*}$ Corresponding author. Tel.: +32-10-478042/472350; fax: +32-10-472180.

E-mail addresses: pierre.vandergheynst@epfl.ch (P. Vandergheynst), antoine@fyma.ucl.ac.be (J.-P. Antoine), anne.goldberg@ solvay.com (A. Goldberg), doghri@mema.ucl.ac.be (I. Doghri).

${ }^{1}$ Present address: Signal Processing Laboratory, EPFL, Lausanne, Switzerland.
} 




Fig. 1. Schematic drawing of the falling-weight system.

scale and is thus essentially transient. Nevertheless, oscillations appear in the force vs. time curve, giving the impression that high frequency phenomena happen during this very rapid test.

Because of its particular multiaxial geometry and the specific system dynamics, many problems of interpretation of an IFWI test still exist, especially in the case of very brittle or very ductile behavior.

In the first case, large dynamic oscillations appear, that can make the recorded signal completely unreadable. The only solution is then to modify the test conditions. On the other hand, in the case of very ductile breaks, it is sometimes very difficult to appreciate the localization of the yield and of the rupture.

In the work reported here, we have chosen two kinds of impact signals: one produced by a rebound (without damage) and one given by a single rupture (one crack) on a clamped PMMA disk. Two representative examples are presented in Fig. 2, and will be used throughout the work.

In order to distinguish between the frequencies due to the equipment and those due to the material, we need a method of analysis that gives information in frequency and in time simultaneously. Indeed, it is reasonable to expect that frequencies caused by equipment vibrations will be present all along the signal, including parts which are not involved in the impact itself. Furthermore those frequencies will be approximately the same whatever test is performed.

In the literature on impact tests, the signal is usually analyzed with the classical Fourier transform (see, e.g., Maurer and Breuer (1995)). However, because of the transient nature of the signal inherent to the physics of the problem, standard frequency (spectral) methods of analysis, such as Fourier, miss their goal, because they are unable to provide a view of what happens both in frequency and in time. This point is illustrated by Fig. 3, which shows the Fourier spectrum of our two signals. In both spectra, the dominant lines are those at 3900 and $4800 \mathrm{~Hz}$, but we will see in Section 5 that they should be attributed to vibrational 

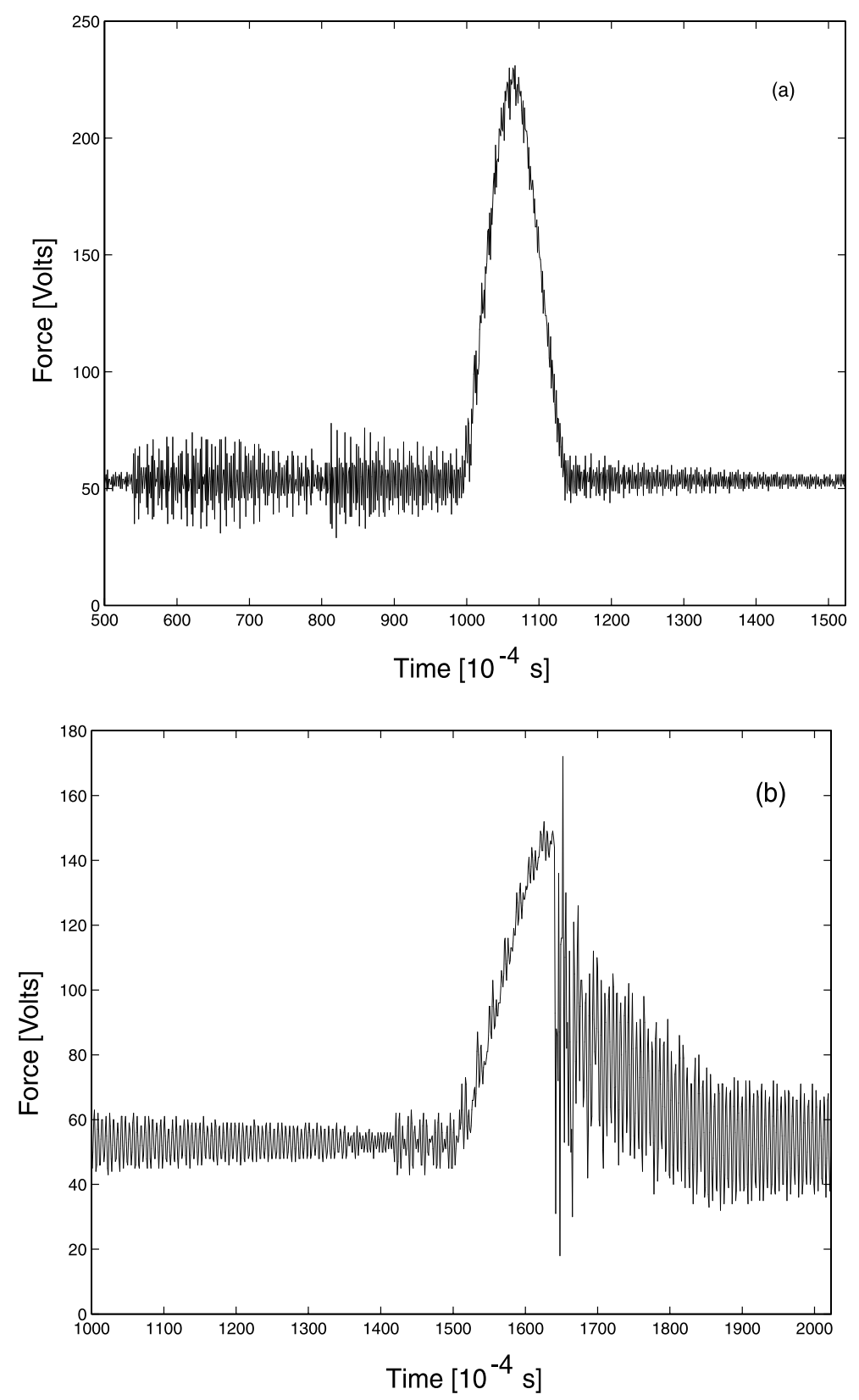

Fig. 2. Typical IFWI results, corresponding to (a) a rebound; and (b) a rupture.

perturbation, not to the impact itself. Other significant lines are visible around 1100, 6800 and $8000 \mathrm{~Hz}$, with less intensity. Actually these lines do correspond to the impact, but their very short duration is not perceptible here. Thus a Fourier analysis is not only inadequate, it is misleading.

In summary, extracting the right information in an IFWI test is still a challenge. In order to solve the problem, we have searched for new, or less classical, methods of signal analysis. It is the aim of the present 



Fig. 3. Fourier power spectra (i.e., $|\widehat{s}(v)|^{2}$ as a function of frequency $v$ ) of the two signals: (a) the rebound; and (b) the rupture.

work to present some original results obtained with the help of some time-frequency methods. Two such techniques are popular nowadays, the short time fourier transform (STFT), also called Gabor transform (GT) (see Feichtinger and Strohmer (1998)), and the continuous wavelet transform (CWT) (see Daubechies (1992), Mallat (1999), Meyer (1992), Torrésani (1995)). We have mostly used the latter, because it is considerably more efficient in detecting and characterizing discontinuities in a signal, like the one we are measuring. Since this technique may not be familiar to the reader, we will start with an overview of the wavelet method. However, when it comes to detecting characteristic frequencies in a signal, the GT is sometimes advantageous, and we have exploited it also, essentially for confirming the results obtained with the wavelet method. In addition, and for the sake of comparison, we have also analyzed our signals with the Wigner-Ville method (see Mecklenbräuker and Hlawatsch (1997)). Although heavier to use, this last method offers some advantages in certain cases. 
The signals are perfectly readable without any mathematical method, so that the results can be interpreted in an objective way. The goals of the work are:

- to identify the moment of rupture, if any;

- to determine the vibration modes excited during impact;

- to determine and eliminate frequencies due to the equipment and to noise;

- to simulate the impact testing by modal analysis and resolution of the problem of forced vibrations by (i) an analytical method, and (ii) finite element (FE) modeling, and then make a comparison with the experiments.

Altogether, we have analyzed the same two signals (one from an impact with rebound, the other for a case of rupture) with four different methods: standard Fourier analysis, CWT, GT and Wigner-Ville transform. As far as we know, such a comprehensive study has not been performed before, neither for mechanical applications, nor in signal analysis in general.

A successful signal analysis needs the right combination of research and experience. For instance, in order to detect and to characterize the singularities of a signal, it is advantageous to use as analyzing wavelet a derivative of the Gaussian, but the order of the derivative has to be determined. If the signal is relatively well defined in frequency, it is more appropriate to use analyzing wavelets which are well localized in the frequency space. The standard choice is the Morlet wavelet, but one needs to find the optimal choice of parameters. Another example is the choice of the window in the GT, since the results are rather sensitive to this choice. And finally, we note that the efficiency of the smoothened pseudo-Wigner-Ville distribution comes at a price: it is heavy to use and difficult to interpret.

The paper is organized as follows: After a rather extensive summary of the two main time-frequency methods, the CWT and the GT (Section 2), we present the experimental setup (Section 3). Then we perform a first wavelet analysis designed for a precise determination of the instant of impact (Section 4). Next we turn in Section 5 to the description of the frequency behavior during the impact, both by wavelets and Gabor analysis (we also show the corresponding Fourier spectrum, in order to emphasize its shortcomings). Finally, Section 6 is devoted to modal and stress analyses of IFWI experiments, by closed-form solutions and FE models. For the convenience of the reader, we present in Appendix A some mathematical results regarding ridges and skeleton in CWT, and we recall in Appendix B the main assumptions of two plate theories.

\section{Time-frequency representations of signals}

\subsection{The continuous wavelet transform}

The CWT is a mathematical tool that consists in decomposing locally a signal into elementary contributions localized on different scales, called wavelets. It has become a common tool in signal processing with a very wide spectrum of applications. We shall give here an overview of the definitions and the main mathematical properties of the CWT. Further details may be found in textbooks like Daubechies (1992), Mallat (1999), Meyer (1992), Torrésani (1995), or conference proceedings like Meyer (1991), Meyer and Roques (1993).

The wavelets are obtained from a single function $\psi$, the mother wavelet, by translation and dilation,

$$
\psi_{(b, a)}(t)=\frac{1}{\sqrt{a}} \psi\left(\frac{t-b}{a}\right),
$$

where the parameters of translation, $b \in \mathbb{R}$, and dilation, $a>0$, may be continuous or discrete. 
Given a finite energy signal $s(t) \in L^{2}(\mathbb{R}, \mathrm{d} t)$, i.e. $\int_{-\infty}^{+\infty}|s(t)|^{2}<+\infty$, its CWT with the analyzing wavelet $\psi$ is the projection $\left\langle\psi_{(b, a)} \mid s\right\rangle$ of $s$ on the wavelet $\psi_{(b, a)}$. Thus, it is also the convolution of $s$ with a scaled and conjugated wavelet $\psi_{a}^{\#}(t)=\overline{\psi(-t / a)} / \sqrt{a}$ :

$$
S(b, a)=\left\langle\psi_{(b, a)} \mid s\right\rangle=\left(\psi_{a}^{\#} * s\right)(b)=\frac{1}{\sqrt{a}} \int_{-\infty}^{+\infty} \overline{\psi\left(\frac{t-b}{a}\right)} s(t) \mathrm{d} t .
$$

In the Fourier domain, expression (2.2) takes the following form:

$$
S(b, a)=\sqrt{a} \int_{-\infty}^{+\infty} \bar{\psi}(a \omega) \widehat{s}(\omega) \mathrm{e}^{\mathrm{i} \omega b} \mathrm{~d} \omega,
$$

where $\widehat{s}$ and $\widehat{\psi}$ are the Fourier transforms of the signal $s$ and of the wavelet $\psi$, respectively.

The CWT conserves the energy of the signal, namely

$$
\int_{-\infty}^{+\infty} \mathrm{d} b \int_{0}^{+\infty} \frac{\mathrm{d} a}{a^{2}}|S(b, a)|^{2}=c_{\psi} \int_{-\infty}^{+\infty}|s(t)|^{2} \mathrm{~d} t,
$$

where

$$
c_{\psi} \equiv 2 \pi \int_{-\infty}^{+\infty}|\widehat{\psi}(\omega)|^{2} \frac{\mathrm{d} \omega}{|\omega|} .
$$

The analyzing wavelet $\psi$ is assumed to be well localized both in the time domain and in the Fourier domain (while respecting, of course, the Fourier uncertainty relations). If we require, in addition, the wavelet $\psi$ to satisfy the so-called admissibility condition $c_{\psi}<\infty$, then the CWT may be inverted exactly and the original signal may be reconstructed from its wavelet transform:

$$
s(t)=c_{\psi}^{-1} \int_{-\infty}^{+\infty} \mathrm{d} b \int_{0}^{+\infty} \frac{\mathrm{d} a}{a^{2}} \psi_{(b, a)}(t) S(b, a) .
$$

This relation may also be interpreted, as announced, as an expansion of the signal into the wavelets $\psi_{(b, a)}$, with wavelet coefficients $S(b, a)$. Note that a necessary (and almost sufficient) condition for admissibility is that the wavelet have zero mean:

$$
\widehat{\psi}(0)=0 \quad \Longleftrightarrow \quad \int_{-\infty}^{+\infty} \psi(t) \mathrm{d} t=0 .
$$

In addition, one can impose more vanishing moments:

$$
\int_{-\infty}^{+\infty} t^{n} \psi(t) \mathrm{d} t=0, \quad n=1, \ldots, n_{0},
$$

and this in turn ensures that the CWT will filter out any polynomial behavior up to degree $n_{0}$. For instance, the case $n_{0}=1$ means that the CWT is blind to any linear behavior, i.e. it erases any linear trend in the signal. In general, if the wavelet has vanishing moments, the smoother parts of the signal will have very small wavelet coefficients, whereas sharp, nonstationary behavior will give rise to local maxima of the modulus of $S(b, a)$. This explains why wavelet analysis is particularly efficient for the detection of transients.

Eqs. (2.2) and (2.3) show that wavelet analysis is a time-frequency analysis, or, more properly, a timescale analysis (the scale parameter $a$ behaves as the inverse of a frequency). In particular, relation (2.3) shows that the CWT acts as a filter, at constant relative bandwidth $\Delta \omega / \omega=$ constant. As a consequence, the CWT is particularly efficient for detecting and characterizing singularities in a signal.

The main virtues of the CWT follow from the support properties of $\psi$. Assume $\psi$ and its Fourier transform $\widehat{\psi}$ to be as well localized as possible, namely, $\psi$ is negligible outside of a time interval of width $T$, 
centered around 0 , while $\widehat{\psi}$ has an essential support in frequency of width $\Omega$, centered around $\omega_{0}$. Then the transformed wavelets $\psi_{(b, a)}$ and $\widehat{\psi_{(b, a)}}$ have, respectively, $\operatorname{supp} \psi_{(b, a)} \sim a T$ around $b$ and $\operatorname{supp} \widehat{\psi_{(b, a)}} \sim \Omega / a$ around $\omega_{0} / a$. Notice that the product of the two widths is constant (we know it has to be bounded below by a fixed constant, by Fourier's theorem, also known as the uncertainty principle!). Therefore:

- if $a \gg 1, \psi_{(b, a)}$ is a wide window, whereas $\widehat{\psi_{(b, a)}}$ is very peaked around a small frequency $\omega_{0} / a$ : this transform will be most sensitive to low frequencies.

- if $a \ll 1, \psi_{(b, a)}$ is a narrow window and $\widehat{\psi_{(b, a)}}$ is wide and centered around a high frequency $\omega_{0} / a$ : this wavelet has a good localization capability in the time domain and is mostly sensitive to high frequencies.

Combining now these localization properties with the zero mean condition (2.7) and the fact that $\psi_{(b, a)}$ acts like a filter (convolution), we see that the CWT performs a local filtering, both in time and in scale. This means, the WT $S(b, a)$ is nonnegligible only when the wavelet $\psi_{(b, a)}$ matches the signal, that is, the WT filters that part of the signal, if any, that lives around the time $b$ and the scale $a$. In addition, the discussion above shows that the CWT filter works at constant relative bandwidth $\Delta \omega / \omega=$ constant.

At this point we ought to emphasize that the CWT described here, even discretized for practical reasons, is totally different from the so-called discrete wavelet transform (DWT). The latter is based on the notion of multiresolution analysis and often leads to the construction of orthogonal bases of wavelets, which is almost never the case for the CWT. Also the two approaches have different aims. While the CWT is a tool for analysis and feature determination, as will be seen in the present paper, the DWT is the preferred technique for data compression and signal synthesis, and the most popular one in the signal processing community.

By construction, there is one CWT for each choice of the analyzing wavelet $\psi$ and, in all cases, the CWT has the generic properties described above. For each application, one should select an analyzing wavelet $\psi$ adapted to the type of signal at hand. For instance, in order to detect and to characterize the singularities of a signal or a curve, it is advantageous to use as analyzing wavelet a derivative of a smoothing function $\phi$, namely $\psi(t)=\frac{\mathrm{d}^{n}}{\mathrm{~d} t^{n}} \phi(t)$. Indeed, upon inserting this definition in Eq. (2.2), and assuming $\phi$ regular enough, the CWT of a typical signal reads

$$
S(b, a)=\left(\phi_{a}^{\#} * \frac{\mathrm{d}^{n} s}{\mathrm{~d} t^{n}}\right)(b)=\frac{\mathrm{d}^{n}}{\mathrm{~d} b^{n}}\left(\phi_{a}^{\#} * s\right)(b),
$$

where $\phi_{a}^{\#}(t)=\overline{\phi(-t / a)} / \sqrt{a}$. Eq. (2.9) shows that $S(b, a)$ is simply the $n$th derivative of the signal blurred at resolution (scale) $a$. Thus we expect large wavelet coefficients to occur at locations of sharp variation of $s$. The most common of such wavelets are successive derivatives of a Gaussian,

$$
\psi_{(n)}(t)=\frac{\mathrm{d}^{n}}{\mathrm{~d} t^{n}} \mathrm{e}^{-t^{2} / 2 \sigma^{2}}
$$

where the width $\sigma$ must be adjusted in order to get the best resolution. In the present paper, since we are analyzing sharp singularities, we shall use the two simplest cases, namely $\psi_{(1)}(t)$ and $\psi_{(2)}(t)$, the first and second derivatives of a Gaussian (the latter is called the Mexican hat wavelet).

On the other hand, if the signal is relatively well defined in frequency, as in spectroscopy (spectral lines), it is more appropriate to use analyzing wavelets which are well localized in the frequency space. This is the case of the Morlet wavelet, defined by

$$
\psi(t)=\mathrm{e}^{\mathrm{i} \omega_{0} t} \mathrm{e}^{-t^{2} / 2 \sigma_{0}^{2}}+\eta(t), \quad \widehat{\psi}(\omega)=\sigma_{0} \mathrm{e}^{-\sigma_{0}^{2}\left(\omega-\omega_{0}\right)^{2} / 2}+\widehat{\eta}(\omega),
$$

where the presence of the correction term $\eta$ is necessary to enforce the admissibility condition. If the product $\omega_{0} \sigma_{0}$ is sufficiently large (typically $\omega_{0} \sigma_{0}>5.5$ ), then the correction term is numerically negligible.

One last remark concerns the implementation of the CWT. Eq. (2.3) means that the wavelet transform $S(b, a)$ is the inverse Fourier transform of a pointwise product of the scaled wavelet filter and the signal. 
This allows to use a regular FFT-based algorithm to compute Eq. (2.3), thus reducing the computational cost of the CWT to $\mathcal{O}(\mathrm{kN} \log N)$ where $N$ is the number of samples of the signal and $k$ is the number of scales used.

In general, one plots the modulus of the wavelet transform $S(b, a)$ as a function of scale $a$ and time $b$. We give an example in Fig. 4, where we show the WT of two typical signals. On the left, a linear chirp is analyzed with a Morlet wavelet, whereas on the right, a discontinuous signal is analyzed with the first derivative of a Gaussian. In this graph, and in all the further ones, our conventions are the following: the graph represents the modulus of the CWT of the signal, with time $b$ on the horizontal axis, and the logarithm of the scale, $\log a$, on the vertical axis, pointing downwards. Thus small scales, i.e., high frequencies, are on the top of the graph.

(a)

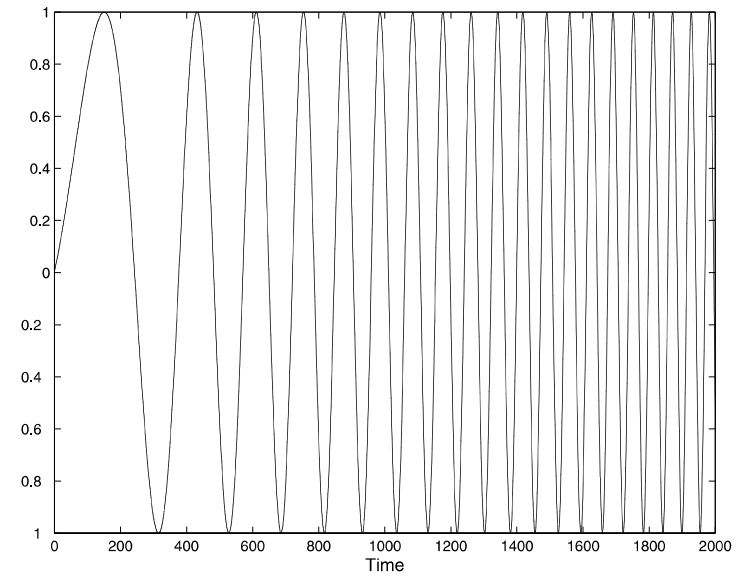

(c)



(b)

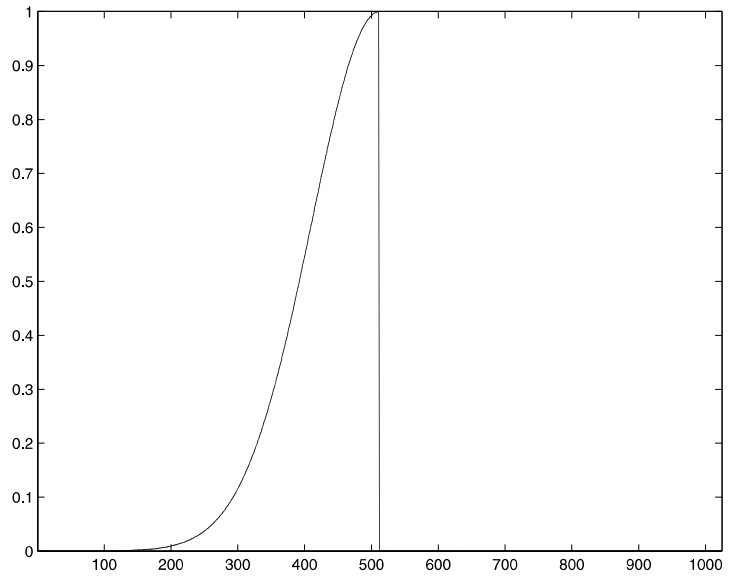

(d)

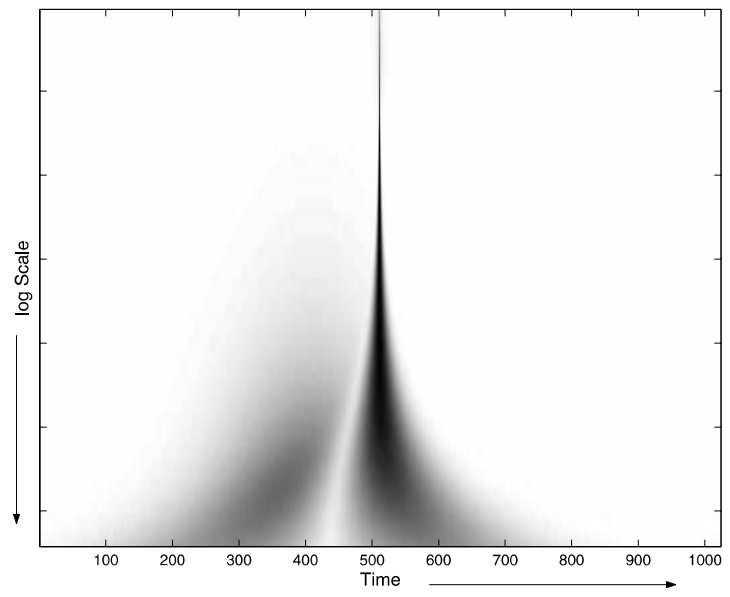

Fig. 4. Examples of wavelet transforms: the top row shows the signals, the bottom row the modulus of the corresponding CWT, in levels of gray. Time $b$ is on the horizontal axis, and the logarithm of the scale, $\log a$, or, equivalently, the frequency $(\sim 1 / a)$, on the vertical axis. Left: a linear chirp, analyzed with a Morlet wavelet; Right: a discontinuous signal, analyzed with the first derivative of a Gaussian. 
However, the resulting picture is often noisy and irrelevant details mask the essential information. For that reason, signal analysts generally use the skeleton of the CWT instead of its modulus. This object is defined as the set of ridges $S_{j}(b, a)$, which are essentially lines of local maxima. It may be shown (see Delprat et al. (1992)) that the restriction of the WT $S(b, a)$ to its skeleton contains the whole information. Thus it is not necessary to compute the whole CWT, but only its skeleton, and this is of course much less costly computationally. Further details may be found in Appendix A.

\subsection{The Gabor and Wigner-Ville transforms}

Alternatively, one may use the STFT, also called GT. The latter consists also in projecting a signal on a family of elementary contributions, obtained from a single window function $g$ by translation and modulation,

$$
g_{(b, \omega)}(t)=\mathrm{e}^{\mathrm{i} \omega(t-b)} g(t-b),
$$

where the parameters of translation, $b \in \mathbb{R}$, and modulation, $\omega \in \mathbb{R}$, may be continuous or discrete. Thus, given a finite energy signal $s(t) \in L^{2}(\mathbb{R}, \mathrm{d} t)$, instead of Eqs. (2.2) and (2.3), its GT with the window $g$ is the inner product of $g_{(b, \omega)}$ with the signal:

$$
\begin{aligned}
G_{s}(b, \omega) & =\int_{-\infty}^{+\infty} \mathrm{e}^{-\mathrm{i} \omega(t-b)} \overline{g(t-b)} s(t) \mathrm{d} t \\
& =\int_{-\infty}^{+\infty} \overline{\widehat{g}(\xi-\omega)} \widehat{s}(\xi) \mathrm{e}^{\mathrm{i} \xi b} \mathrm{~d} \xi
\end{aligned}
$$

In these relations, $g$ is a window function and any square integrable function is admissible. The most frequent choice is simply a Gaussian. Relation (2.13) shows that the GT also acts as a filter, this time at constant bandwidth $\Delta \omega=$ constant. As a consequence, it is mostly efficient for detecting characteristic frequencies in a signal, instead of singularities.

The most common window is the Gaussian window:

$$
g(t)=\mathrm{e}^{-t^{2} / 2 \sigma_{0}^{2}}, \quad \widehat{g}(\omega)=\sigma_{0} \mathrm{e}^{-\sigma_{0}^{2} \omega^{2} / 2} .
$$

We can notice that, with this choice, the CWT $S(b, a)$ calculated with the Morlet wavelet (2.11) and the GT $G_{s}(b, \omega)$ are equal for $a=\omega_{0} / \omega$, which confirms that, in the wavelet case, $1 / a$ plays the role of a frequency.

The main advantages of the GT are:

1. This transform is covariant under frequency translation, i.e., modulation, whereas the wavelet transform is not. In other words, the GT of a modulated signal is equal to a frequency translation of the GT of the signal.

2. The bandwidth of the analyzing window $g_{b, \omega}$ does not depend on the analyzed frequency: the GT is a filtering process with $\Delta \omega=$ constant.

One drawback of the GT is the rather sensitive dependence on the choice of the window, although the latter may be exploited for controlling the resolution by varying the width of the window. In the Gaussian case (2.15), this means varying the width $\sigma_{0}$.

Exactly as for the CWT, the full information is contained in the skeleton, that is, the restriction of the GT to the set of its ridges (see Antoine et al. (2000)). The latter are defined as in the wavelet case, and they will be preferentially horizontal, as with a Morlet wavelet.

A last possibility for a time-frequency analysis is to use the Wigner-Ville transform or distribution (see Flandrin (1993) and Mecklenbräuker and Hlawatsch (1997)). For a signal $s$, this is the function 


$$
W_{s}(t, v)=\int_{-\infty}^{+\infty} \mathrm{e}^{-2 \pi \mathrm{i} t v} \overline{s(t-\tau / 2)} s(t+\tau / 2) \mathrm{d} \tau .
$$

This transform is entirely intrinsic to the signal, since it does not contain any extra function (wavelet, window) that inevitably influences the result. On the other hand, it is quadratic, which implies the apparition of interference terms whenever the signal is a superposition of two components. In order to minimize these as much as possible, one usually considers, instead of Eq. (2.16), the so-called smoothened pseudoWigner-Ville distribution, namely:

$$
\operatorname{SPW}_{s}(t, v)=\int_{-\infty}^{+\infty} \mathrm{d} b g(t-b) \int_{-\infty}^{+\infty} h(\tau) \mathrm{e}^{-2 \pi \mathrm{i} v \tau} \overline{s(b-\tau / 2)} s(b+\tau / 2) \mathrm{d} \tau .
$$

The two window functions $g$ and $h$ may then be adapted to the signal at hand. We will give an example in Section 5.

\section{Description of the experiments}

Disks of PMMA (Perspex ${ }^{\circledR}$ ) were machined out from sheets of $2.1 \mathrm{~mm}$ thickness at a diameter of 150 $\mathrm{mm}$. The mass density of the material is $\rho=1170 \mathrm{~kg} / \mathrm{m}^{3}$. Isotropic linear elasticity was assumed for PMMA with homogeneous properties. Young's modulus was determined by modal analysis and evaluated at $E=5 \mathrm{GPa}$ around $10^{3} \mathrm{~Hz}$ (since the material is very sensitive to strain rate, we tried to measure $E$ at conditions close to those prevailing during impact). Poisson's coefficient $v$ was taken at its classical value for polymers, namely $v=0.35$.

Impacts on clamped disks were performed on a homemade IFWI equipment (see Fig. 1). The conditions of the test are:

- diameter of the striker $=8.5 \mathrm{~mm}$

- diameter of the support $=135 \mathrm{~mm}$

- mass of the striker, $M=1.16 \mathrm{~kg}$

- impact height, $H=2$ and $3 \mathrm{~cm}$, respectively

Detailed information on the experimental setup for IFWI tests can be found in references such as Savadori (1985) and Maurer and Breuer (1995). The main points are the following.

A servo-hydraulic testing machine is used. A piezo-electric load cell is fixed to the striking dart. The acquisition frequency ranges between 10240 and $20480 \mathrm{~Hz}$ on a Philips oscilloscope. The test's main measures are the impact force $F$ (the signal measured in Volts is later transformed into Newtons) vs. time $t$ and the striker's initial velocity $v_{0}$. From momentum conservation (see Savadori (1985), Van Vyve (1997)) other quantities can be readily computed, e.g., displacement vs. time, force vs. displacement, energy vs. time and energy vs. displacement.

\section{Detection of the singular events in the signal with Gaussian wavelets}

In order to detect the different discontinuities in the two signals of Fig. 2, rebound and rupture, in particular the moment of the rupture in the second case, we analyze them with a first and a second derivative wavelet, namely, the wavelets (2.10), with width $\sigma=1$,

$$
\psi_{(1)}(t)=\frac{\mathrm{d}}{\mathrm{d} t} \mathrm{e}^{-t^{2} / 2}, \quad \psi_{(2)}(t)=\frac{\mathrm{d}^{2}}{\mathrm{~d} t^{2}} \mathrm{e}^{-t^{2} / 2}
$$

Each discontinuity is characterized by a vertical ridge in the wavelet transform. 
For the case of a rebound (no rupture), we present in Fig. 5 the result of the analysis for the two wavelets successively. From top to bottom, the figure shows, as a function of time, the modulus of the CWT, its skeleton, and the characteristic points in the signal detected by the respective ridges. For $n=1$ (left), the CWT detects (in the sense that the ridges point toward) the zero-crossings of the second derivative of the signal, that is, the points of inflection, rather than the discontinuities themselves. The latter correspond to loading and unloading and are perfectly detected in the second case, $n=2$ (right). Moreover, the evolution of the modulus of the wavelet transform as a function of the logarithmic scale shows a very similar shape for both events. Going from small scales to large scales, the modulus first increases, passes through a maximum around $\ln a \simeq 2.75$, then decreases (Fig. 6). The maximum may also be seen directly on the amplitude, in Fig. 5(a) and (b), around $a \simeq 16 \simeq \mathrm{e}^{2.75}$. The discrepancy between the two curves of Fig. 6 is visible at small scales and it may be related to the viscoelastic characteristics of the polymer. We must indeed keep in mind that in such a test, velocity varies considerably, including a passage at zero, and this can affect the behavior of the material, especially its stiffness.

In a second test, we analyze the event with a rupture, using the second order wavelet $\psi_{(2)}$. As can be seen in Fig. 7 (right), we find again a ridge that may be attributed to the loading. At the rupture, a very intense ridge appears, followed by several shorter ones. The respective evolutions of the two main ones are represented in Fig. 8. It can be observed that the modulus of the loading ridge has the same shape as that of the loading in the case of rebound, whereas the first ridge characterizing the rupture has a completely different aspect, and increases when scale decreases. Furthermore, this ridge converges rapidly towards the rupture break. Notice that, if one is only interested in locating the break point, then the first order wavelet $\psi_{(1)}$ is sufficient. However, one then loses the localization of the loading ridge, as can be seen by comparing Fig. 7 (left) with Fig. 2(b).

We may now summarize the results as follows. The beginning of the impact is characterized by a ridge function which increases with increasing scale. The rupture is characterized by a decreasing ridge that converges to the rupture moment at small scales. Detection and localization of the different singularities have thus been realized. Of course, this has yet to be implemented on other testing conditions and other materials.

\section{Frequency analysis with a Morlet wavelet, a Gabor transform and Wigner-Ville distribution}

We are now interested in the frequency information contained in the signal. In order to perform such an analysis, we choose a Morlet wavelet (2.11).

As explained in Appendix A, if the signal consists of a single spectral line,

$$
s(t)=A(t) \sin \Omega t,
$$

where the amplitude $A(t)$ varies slowly, then $|S(b, a)|$ has a maximum for $a^{*}=\omega_{0} / \Omega$, and $\left|S\left(b, a^{*}\right)\right|=r(b)$ is proportional to $A(b)$. This means that in this case, the modulus of the transform will reach a maximum value for a scale exactly proportional to $\Omega^{-1}$.

As usual, the product $\omega_{0} \sigma_{0}$ must be sufficiently large, for the corrective factor to become negligible. In our case, $\omega_{0}$ is set equal to 15 and $\sigma_{0}$ to 2 . Our two signals are analyzed again and the results are presented in Figs. 9 and 10.

On the two top graphs of Fig. 9, we present the modulus and the skeleton of the CWT for the rebound signal of Fig. 2(a), that is, the frequency as a function of time. The two lower graphs show the time evolution of the instantaneous frequency and the modulus along a ridge. The same quantities are shown in Fig. 10 for the rupture signal of Fig. 2(b). We can distinguish several ridges, corresponding to 1100, 3900 and $4800 \mathrm{~Hz}$, respectively.

1. Analysis of the frequencies present at 3900 and $4800 \mathrm{~Hz}$ : Examining the evolution of the modulus as a function of time along those two frequencies (3900 and $4800 \mathrm{~Hz}$ ), it can be seen that they are very erratic 

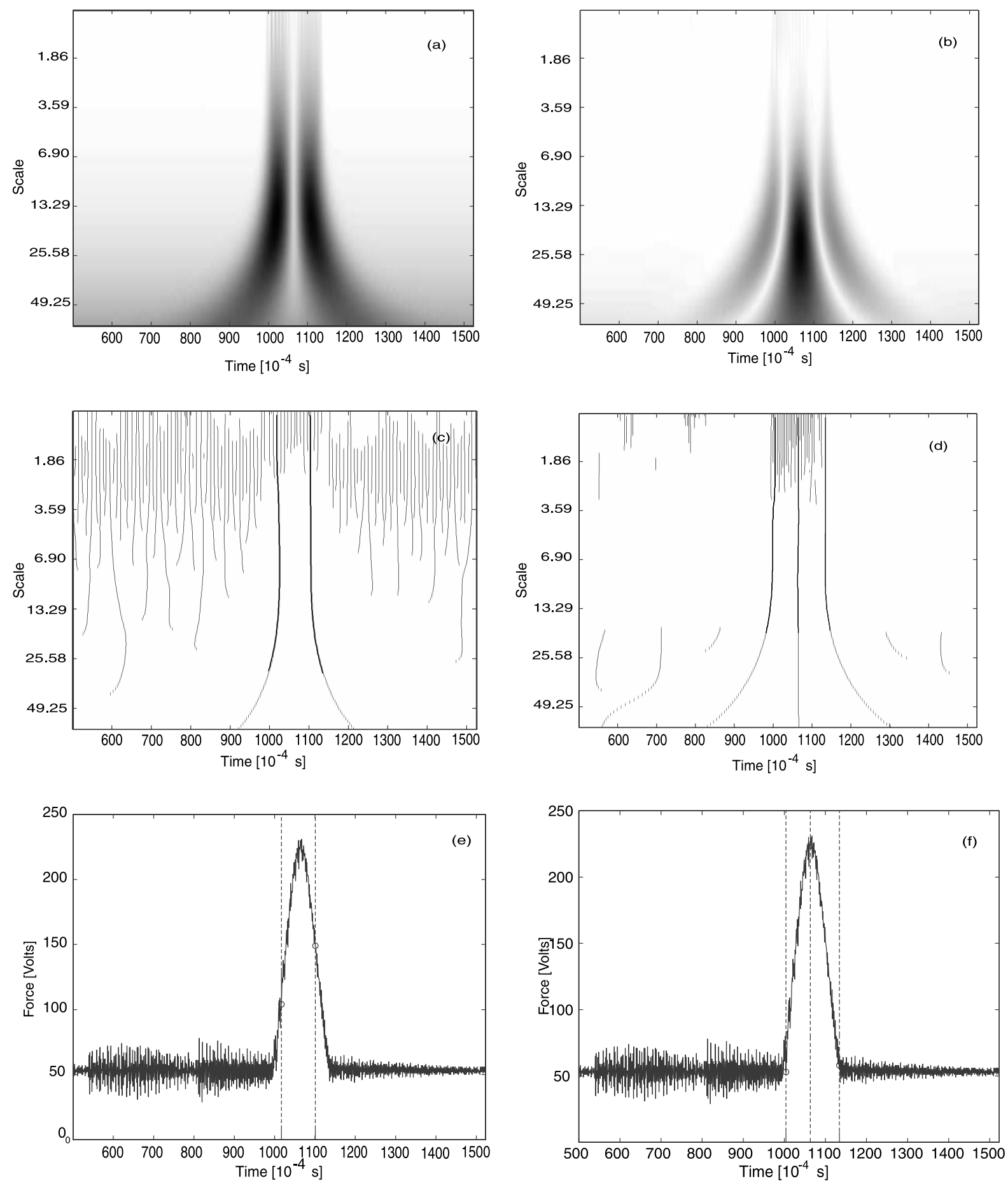

Fig. 5. Analysis of the rebound signal, with the first (left) and second (right) derivative of a Gaussian, respectively: (a,b) the modulus of the CWT; $(\mathrm{c}, \mathrm{d})$ the corresponding skeleton; and $(\mathrm{e}, \mathrm{f})$ the points of the signal detected by the respective ridges. 


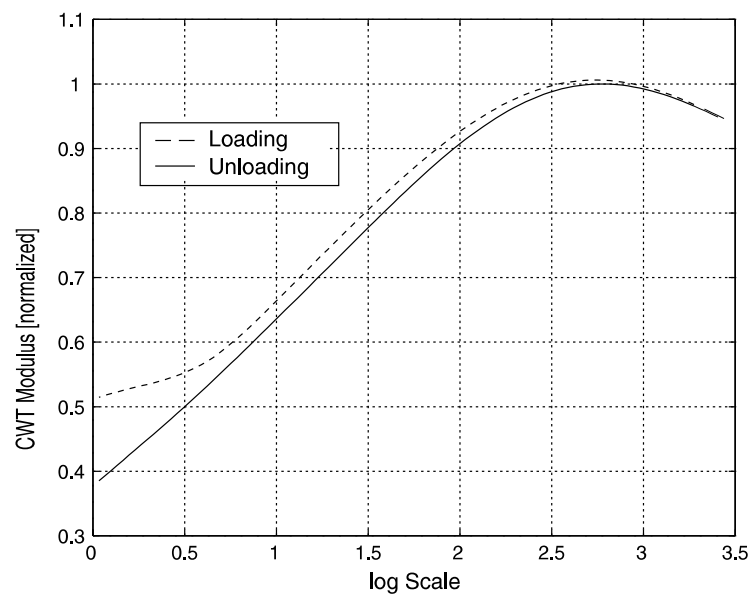

Fig. 6. Rebound: modulus of CWT along the loading (-) and unloading (- - -) ridges.
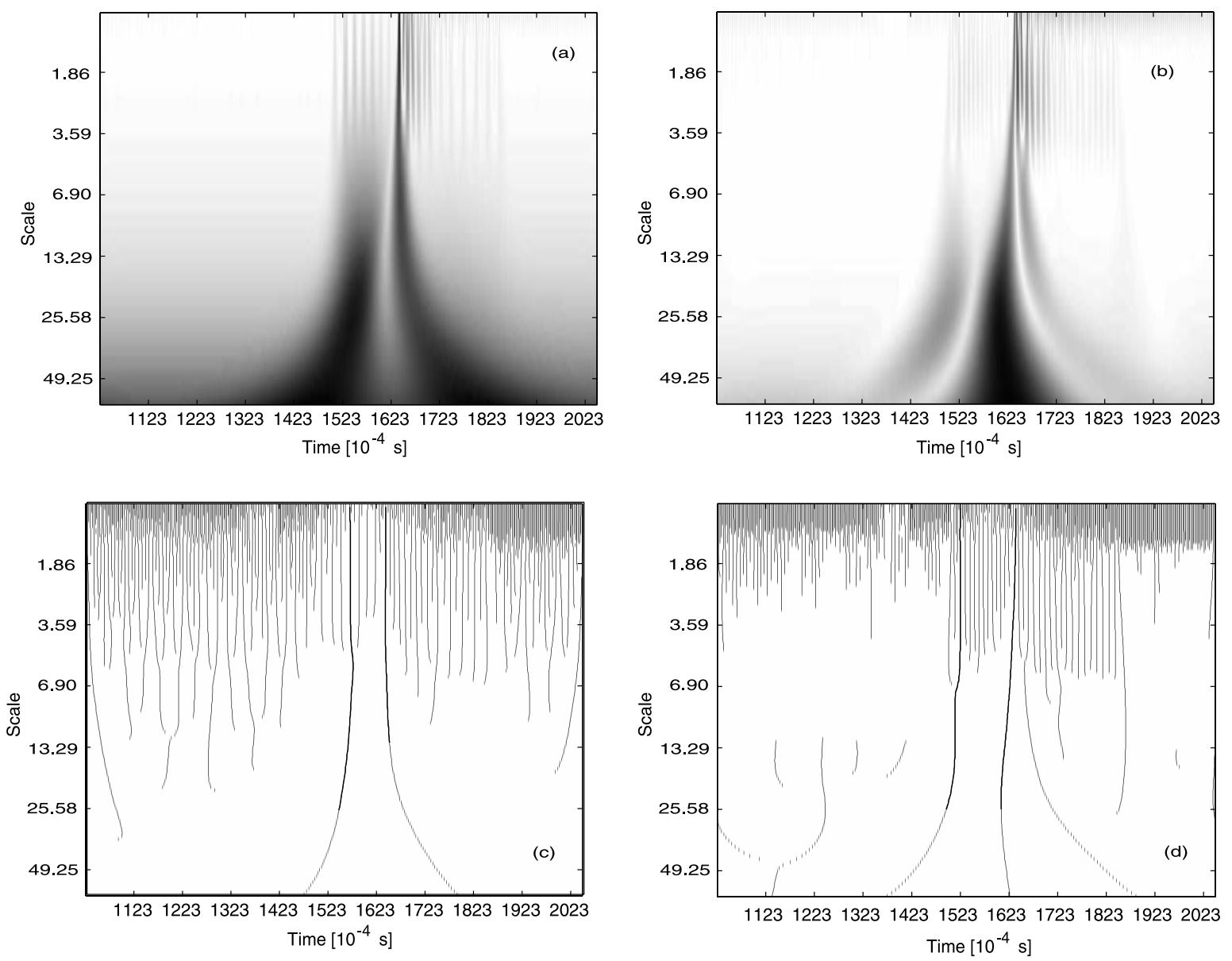

Fig. 7. The case of a sample rupture, analyzed with $\psi_{(1)}$ (left) and $\psi_{(2)}$ (right): (a,b) modulus of the CWT; and (c,d) its skeleton. 

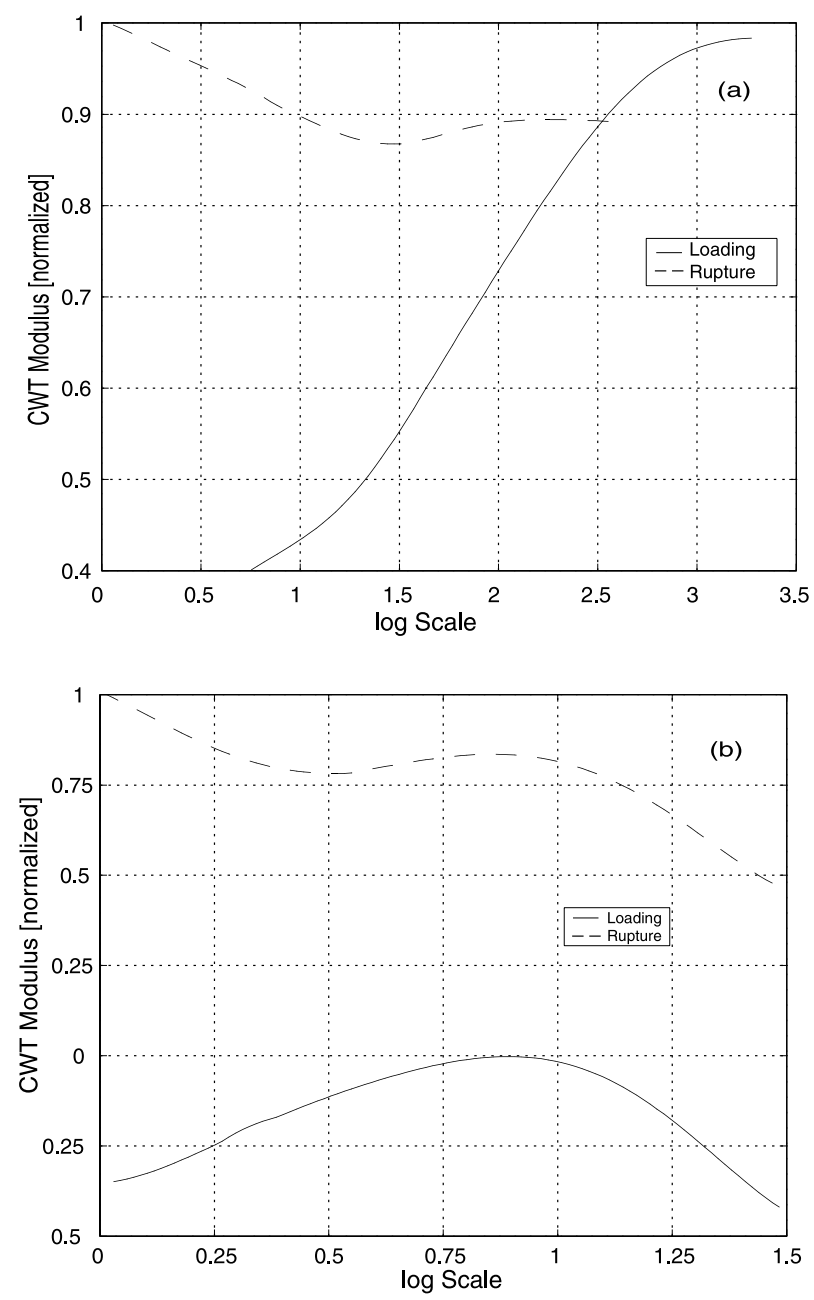

Fig. 8. Sample rupture: modulus of CWT along the two main ridges, one associated to loading (-), and one to the rupture (- - -). The analysis is made successively with (a) $\psi_{(1)}$; and (b) $\psi_{(2)}$.

for both signals, see Van Vyve (1997). Furthermore, they are present all along the signal and their intensity varies with the level of apparent noise present in the signal, which means that they come directly from the equipment.

2. Analysis of the frequency around $1100 \mathrm{~Hz}$ : We can observe the apparition of a frequency that stabilizes around $1100 \mathrm{~Hz}$ and whose intensity is a bell-shaped curve. On the rebound signal, Fig. 9(c) and (d), the value is established to $1115 \mathrm{~Hz}$, the lifetime of this ridge is around $16 \mathrm{~ms}$, which corresponds to the time of the rebound. In the signal with rupture, Fig. 10(c) and (d), this frequency appears around 1025 $\mathrm{Hz}$, and decreases after the rupture to lower values. The total lifetime is also around 17-19 ms. We shall see that those frequencies correspond to a vibration mode of the disk.

3. Other frequencies: Finally, one observes in Fig. 10(b) a last intense ridge around $6800 \mathrm{~Hz}$ in the curve with rupture. This one is also associated with a bell-shaped curve, and occurs just at the moment of the rupture. Its origin is relatively obscure. 



Fig. 9. Time-frequency analysis of the rebound signal of Fig. 2 (a) with a Morlet wavelet: (a) modulus of the CWT; (b) its skeleton; (c) instantaneous frequency along the ridge around $1100 \mathrm{~Hz}$, as a function of time; and (d) modulus of the CWT along that ridge.

As a confirmation of these results, we present in Fig. 11a Gabor analysis of the same two signals. The window is a Gaussian of width $\sigma_{0}=15$. On the rebound signal, Fig. 11(a) and (b), one sees as before:

- a line around $1100 \mathrm{~Hz}$ excited during the time of contact,

- two lines around 3900 and $4800 \mathrm{~Hz}$, present through the whole signal, thus attributable to noise.

Similarly, the same frequencies around 1100, 3900 and $4800 \mathrm{~Hz}$ are seen on the rupture signal, Fig. 11(c) and (d), and, in addition, the two lines around 6800 and $8000 \mathrm{~Hz}$, exactly as with the Morlet wavelet analysis.

One may notice that a single analysis yields all the lines at once, from low to high frequencies, but without the standard correlation between the length of the piece of a signal and the corresponding main frequency (or scale), as seen with the CWT. Thus we see both the advantages and the limitations of the GT with respect to the CWT.

For the sake of comparison, it is instructive to go back to Fig. 3, which shows the Fourier power spectrum of our two signals. Clearly all the previous lines are visible here too, but the method is manifestly 

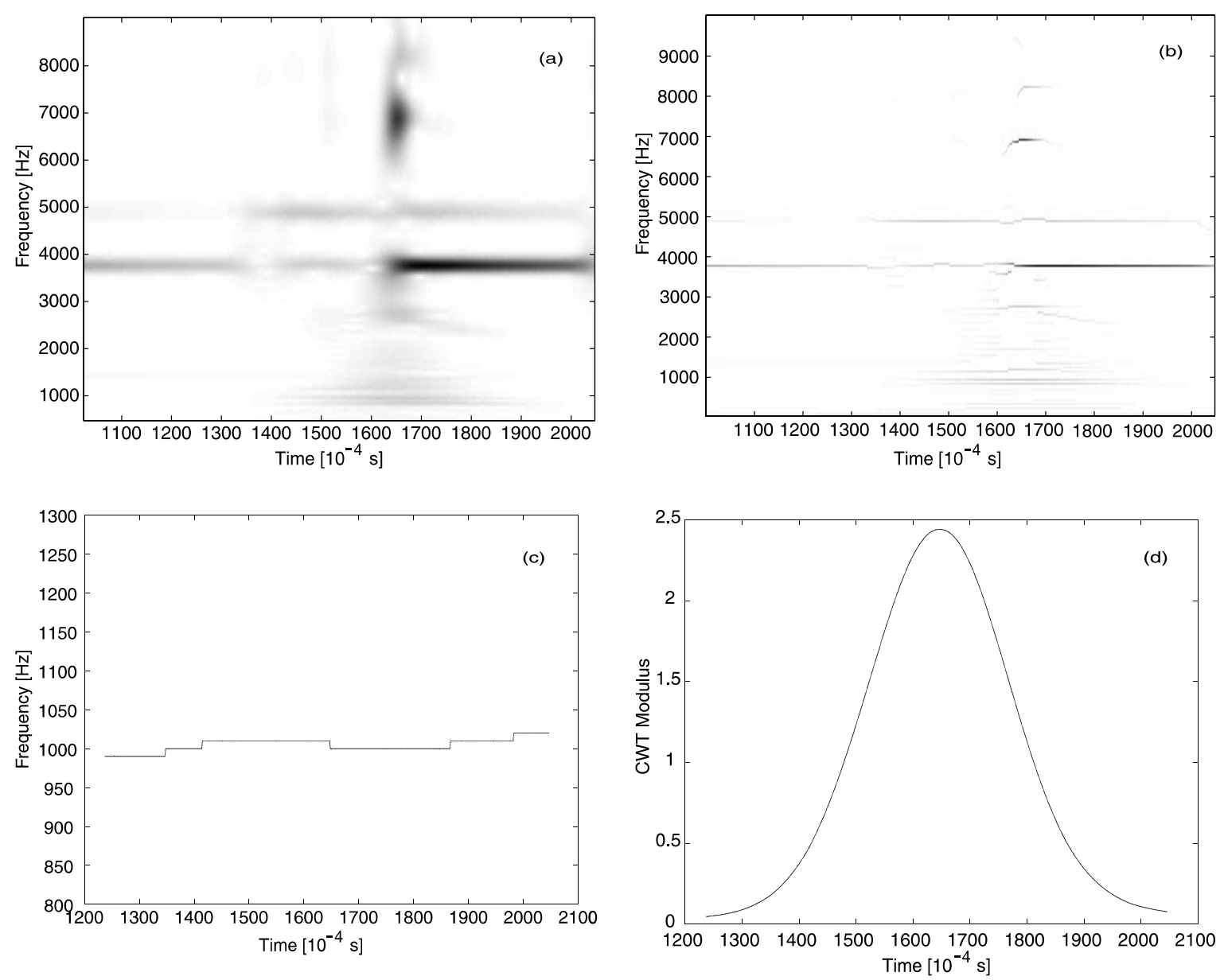

Fig. 10. Time-frequency analysis of the rupture signal of Fig. 2 (bottom) with a Morlet wavelet: (a) modulus of the CWT; (b) its skeleton; (c) instantaneous frequency along the ridge around $1100 \mathrm{~Hz}$, as a function of time; and (d) modulus of the CWT along that ridge.

insufficient, on two counts. First, the dominant lines, in both spectra, are those at 3900 and $4800 \mathrm{~Hz}$, and we have seen that they should be attributed to vibrational perturbation, not the impact itself. Second, the relevant lines, around 1100, 6800 and $8000 \mathrm{~Hz}$, are well visible, albeit with less intensity, but their very short duration is of course not perceptible. Thus a Fourier analysis is totally misleading here. Only a time-frequency method can make the difference between transient events and persistent vibrations, independent of the impact.

In addition, we show in Fig. 12 the analysis of our two standard signals, rebound and rupture, with a smoothened pseudo-Wigner-Ville distribution. Notice that, for better visibility, each Wigner-Ville plot is flanked, above, by the corresponding signal (time picture). In addition, we present on the left the corresponding energy spectral density (frequency picture),

$$
|\widehat{s}(\omega)|^{2}=\int_{-\infty}^{+\infty} S P W_{s}(t, \omega / 2 \pi) \mathrm{d} t
$$



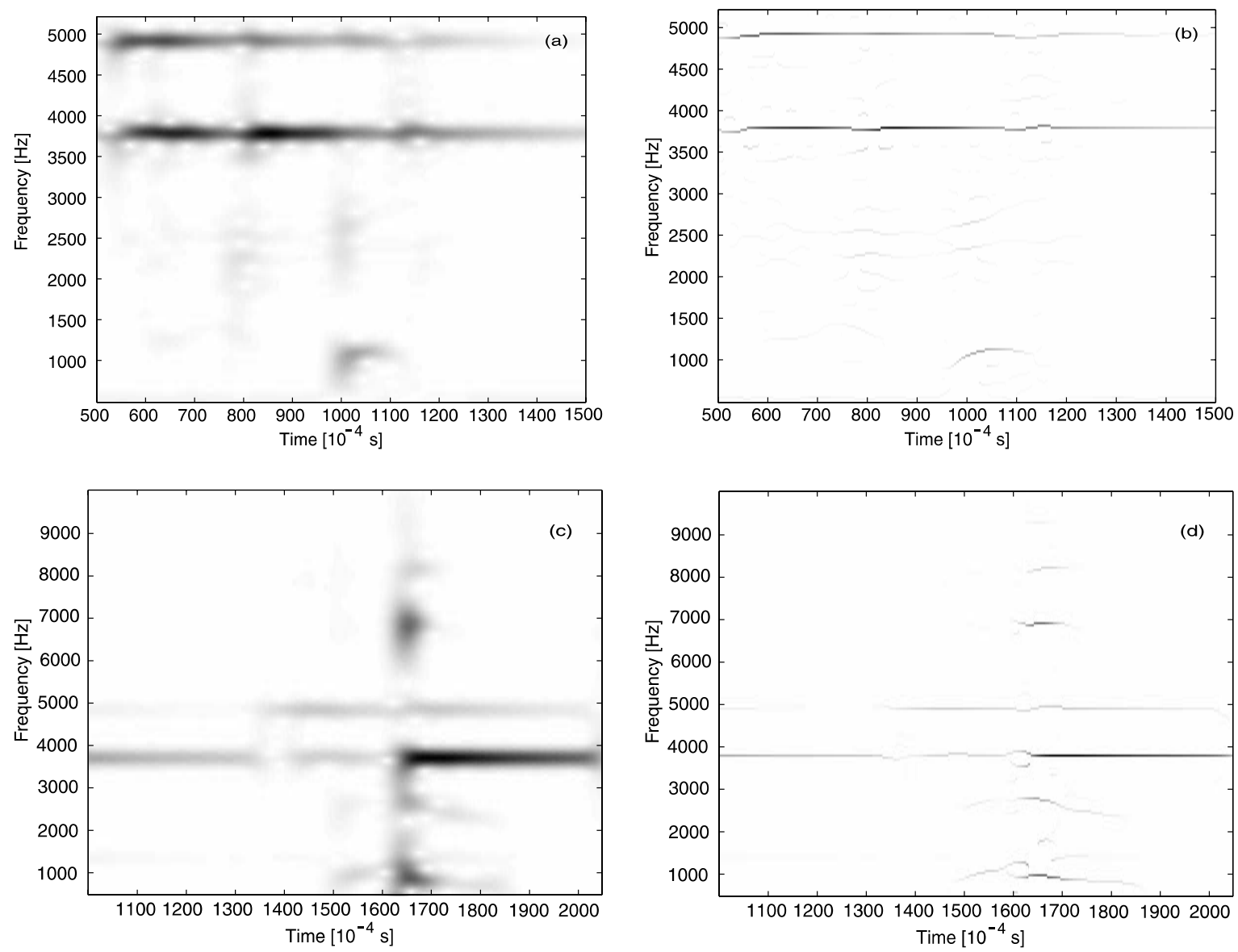

Fig. 11. Gabor analysis of the two signals of Fig. 2 and the corresponding skeletons: (a,b) the rebound signal; (c,d) the rupture signal.

that is, the power spectrum given in Fig. 3. Here again one can recognize all the characteristic frequencies found previously, as well as the points of discontinuity, all in one single figure. Were it not so heavy and difficult to interpret, this technique would be the most efficient one.

\section{Impact test simulation}

Different models were tested in order to simulate the impact test. Both analytical and numerical (using the finite element method (FEM)) analyses were carried out.

\subsection{The analytical model}

An analytical model has been studied, on the basis of the Kirchhoff-Love (KL) theory for bending of thin plates. The main points of the latter are summarized in Appendix B. For a plate clamped at its outer edge $(r=a)$, closed-form solutions were obtained in the following cases: 
(a)


(b)
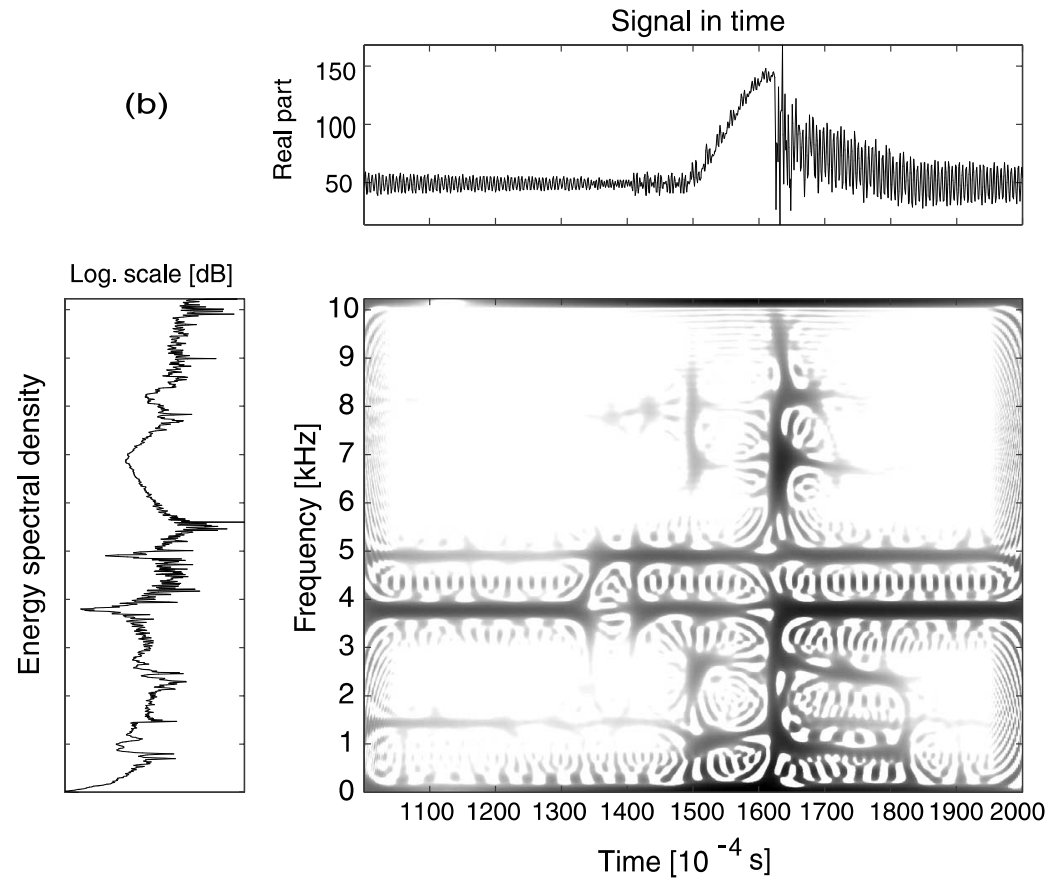

Fig. 12. Smoothened pseudo-Wigner-Ville distribution of the same signals: (a) rebound; (b) rupture. 
(C1) Free vibration (zero pressure: $p=0$ ).

(C2) Forced vibration with axisymmetric load, i.e. a pressure distributed over a concentric disk of radius $r_{0}: p(r, \theta, t)=p(r, t)$ for $r \leqslant r_{0}$ and $p(r, \theta, t)=0$ for $r>r_{0}$. The pressure was assumed to be uniform for simplicity: $p(r, t)=p_{0}(t)$ for $r \leqslant r_{0}$.

(C3) Forced vibration with a point load at $\left(r=b, \theta=\theta_{0}\right)$, i.e. a load which presents an eccentricity with respect to the center of the plate.

The complete derivations are detailed in Van Vyve (1997). The values of the eigenfrequencies $f_{k n}$ (free vibration, $p=0$ ) obtained with the data of Section 3 are presented in Table 1 . The corresponding vibration modes are shown in Fig. 13. Subscripts $k$ and $n$ designate the number of modal diameters and circles, respectively.

Table 1 shows that the value of computed frequencies which is closest to the experimental one $(1050 \mathrm{~Hz})$ is $f_{11}=994 \mathrm{~Hz}$, corresponding to a nonsymmetric mode (one modal diameter and one modal circle).

Forced vibrations (cases (C2) and (C3)) were also studied in Van Vyve (1997). The results show that:

- for an axisymmetric situation, the only eigenmodes are axisymmetric (respiration),

- when the number of modal circles $n$ increases, the displacement amplitude decreases rapidly;

- the value of the displacement decreases with the increase of $r_{0}$ (the radius of the loading zone);

- the presence of an eccentricity does not alter the response in any significant way: nonsymmetric modes remain negligible in front of respiration modes.

\subsubsection{Conclusion of the analytical modeling}

The model yields a symmetrical mode at $500 \mathrm{~Hz}$, but not at $1000 \mathrm{~Hz}$, even when considering an eccentric loading. Furthermore, the comparison between computed and measured displacements shows no agreement between them.

Consequently this model is not adapted to the description of the impact problem, and another type of analysis is needed (see Section 6.2).

\subsection{Finite element computations}

Considering the limited results obtained with the analytical analysis, more realistic models were studied numerically using the FEM. The validity of the different models will be based on a modal analysis, yielding the different frequencies for the vibration. The displacement and stresses in the plate will be computed with

Table 1

Eigenfrequencies $f_{k n}(\mathrm{~Hz})$ derived from Kirchhoff's model ${ }^{\mathrm{a}}$

\begin{tabular}{llllr}
\hline$f_{k n}$ & $n$ & & & \\
\cline { 2 - 5 }$k$ & 1 & 2 & 3 & 7392 \\
\hline 0 & 477 & 1859 & 5611 & 9302 \\
1 & 994 & 2843 & 7188 & 11343 \\
2 & 1630 & 3953 & 8893 & 13514 \\
\hline
\end{tabular}

${ }^{\mathrm{a}} k$ and $n$ : number of modal diameters and circles, respectively. 



Fig. 13. The first three vibration modes of the clamped plate.

the most adapted model, and a classical fracture criterion will be applied. For a presentation of the FEM, see e.g., Hughes (1987).

\subsubsection{Finite element modeling}

FE simulations were obtained for two plate elements: KL and Reissner-Mindlin (RM); see Hughes (1987) for more information. The RM plate theory allows for transverse shear (see Appendix B).

The results were first obtained in Van Vyve (1997) with the SAMCEF program [SAMCEF (1998)]. Later, the same problems were run again in Risse (1998) with the ABAQUS package [ABAQUS (1992)].

The complete geometry was meshed. Axisymmetry was not assumed for two reasons. First, non-symmetric modes and not only respiration (axisymmetric) modes are sought. Second, with a complete model, the influence of eccentricity can be analyzed further.

The mesh which was used in all simulations consisted of 600 four-noded plate elements: 20 in the radial direction and 30 in the hoop direction. It is shown in Fig. 14.

One of the main reasons for the shortcomings of the analytical procedures of Section 6.1 is that the inertia of the striker was not taken into account. However, experimental data show that the striker's mass $(1.16 \mathrm{~kg})$ is 33 times larger than that of the plate $(35.2 \mathrm{~g})$. Therefore, neglecting the inertia of the striker is a bad assumption. In the FE simulations, we consider that the mass of the striker is uniformly distributed over a central disk of radius $r_{0}=1 \mathrm{~mm}$.

\subsubsection{Modal analysis results obtained with different models}

Frequency results were obtained for different numerical simulations and are reported in Table 2. The Lanczos method was used for eigenvalue extraction, as explained in the theory manual of ABAQUs and in Hughes (1987). 


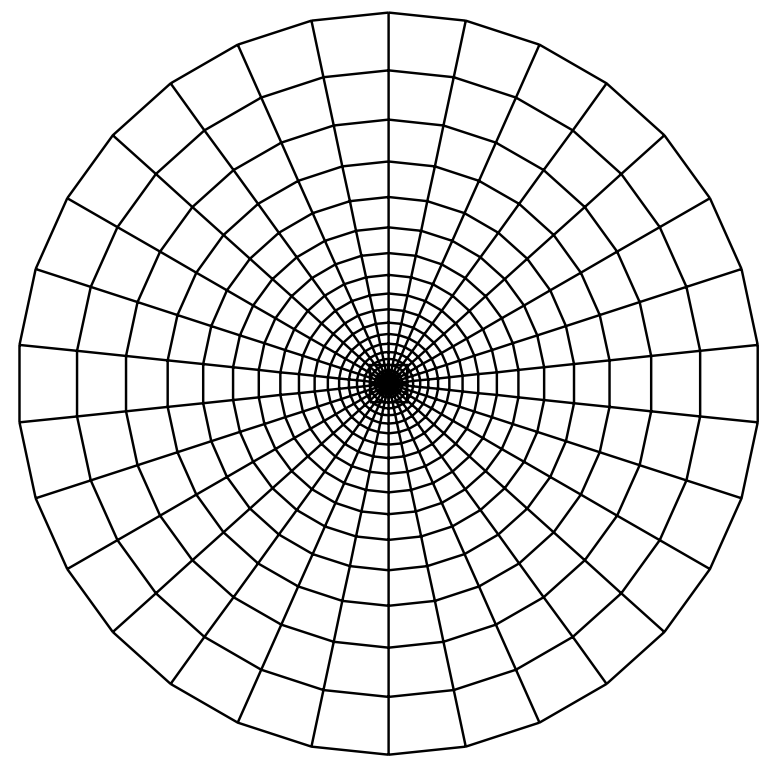

Fig. 14. The FE mesh.

Columns 2 and 3 of Table 2 correspond to FE results using KL and RM plate elements, respectively. In both cases, only the impact force was taken into account, this is in order to validate the FE model against the analytical results which are reported in column 1 . The results show a good agreement between analytical and FE results, which gives confidence in the FE model which was built.

Column 4 of Table 2 gives FE results for RM plate elements in the case where the inertia of the striker is taken into account as explained in Section 6.2.1, i.e., by uniformly distributing the striker's mass over a central disk of radius $r_{0}=1 \mathrm{~mm}$.

Comparison of columns 1-4 also shows that when the striker's mass is taken into account (column 4), the values of the frequencies decrease steeply by comparison to the no-inertia cases (columns 1-3), especially the second respiration mode $\left(f_{02}\right)$. That value corresponds to the one which was found using wavelet analysis in Section 5.

Numerical results reported in Van Vyve (1997) and Risse (1998) show that the values of the frequencies for nonsymmetric modes (e.g., $f_{11}$ ) vary significantly with the value $r_{0}$ which is assigned to the contact area radius, while the values of symmetric (or respiration) modes (e.g., $f_{02}$ ) are almost insensitive to a variation of $r_{0}$.

Table 2

Modal analysis with different models (Eigenfrequencies $f_{k n}(\mathrm{~Hz}), k$ and $n$ : number of modal diameters and circles, respectively)

\begin{tabular}{|c|c|c|c|c|}
\hline & Kirchhoff (analytical) & Kirchhoff & Mindlin & Mindlin with mass \\
\hline$f_{01}$ & 477 & 481 & 475 & 32 \\
\hline$f_{11}$ & 994 & 1001 & 984 & 672 \\
\hline$f_{21}$ & 1630 & 1642 & 1598 & 1084 \\
\hline$f_{02}$ & 1859 & 1872 & 1839 & 1050 \\
\hline$f_{31}$ & 2385 & 2403 & 2301 & 1499 \\
\hline
\end{tabular}




\subsubsection{Displacements and stresses}

The plate and striker system is considered again, with the striker's mass uniformly distributed within a central zone of radius $r_{0}=1 \mathrm{~mm}$. In addition, the impact is modeled in a simplified manner by giving an initial velocity $v_{0}=0.767 \mathrm{~m} / \mathrm{s}$ (that of the striker) to the nodes of the central zone.

The discretized weak equations of the dynamical problem are numerically integrated in time using the Hilber-Hughes-Taylor algorithm; see the ABAQus theory manual and Hughes (1987) for details. A sufficiently small time step was chosen $(0.1 \mathrm{~ms})$.

The displacement and stress fields were computed for the rebound experiment with RM plate elements. Comparison between computed and measured displacements at the center of the disk shows (Fig. 15) that:

1. The extreme values are quasi-identical: $3.79 \mathrm{~mm}$ at the maximum $(t=7.9 \mathrm{~ms})$ and $0 \mathrm{~mm}$ after $15.5 \mathrm{~ms}$.

2. The computed curve is completely symmetric, which is not the case in the experiment. Note that numerical results for time $>15.5 \mathrm{~ms}$ (i.e., when the displacement becomes positive) are not reliable, because there is a loss of contact between the striker and the plate which is not taken into account by the FE model.

Inside the loading zone, the radial and hoop stresses are equal $\left(\sigma_{r r}=\sigma_{\theta \theta}\right.$ for $\left.r \leqslant r_{0}\right)$, while outside the zone, the hoop stress is mostly tensile and larger than the (mostly tensile) radial stress. Fig. 16 shows the time evolution of the stress at $(r=0, z=h / 2)$.

Fig. 17 shows the spatial distribution of $\sigma_{r r}$ and $\sigma_{\theta \theta}$ at time $t=7.9 \mathrm{~ms}$, which corresponds to the maximum displacement.

It can be seen from Fig. 16 that the maximum tensile stress (115 MPa) occurs at $t=7 \mathrm{~ms}<7.9 \mathrm{~ms}$. The spatial stress distribution (Fig. 17) shows that the tangential stress is higher than the radial one, which

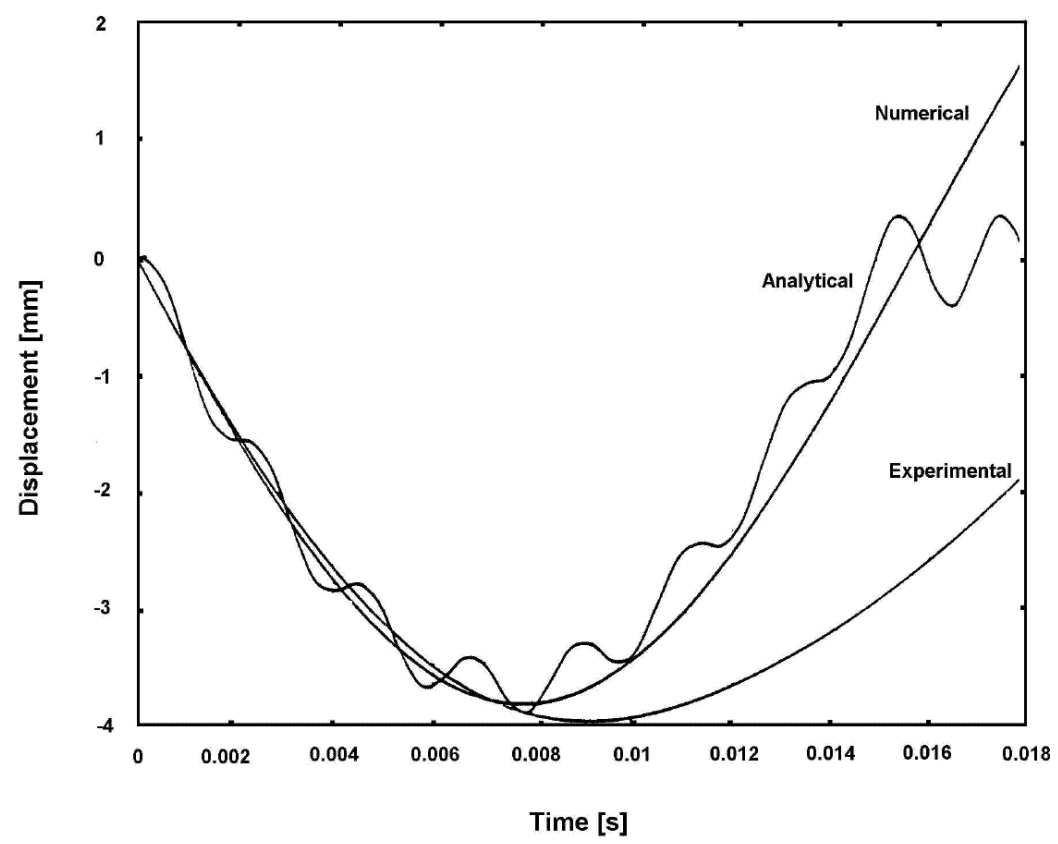

Fig. 15. Displacement (mm) of the center node vs. time (s). Experimental, numerical and analytical results. 


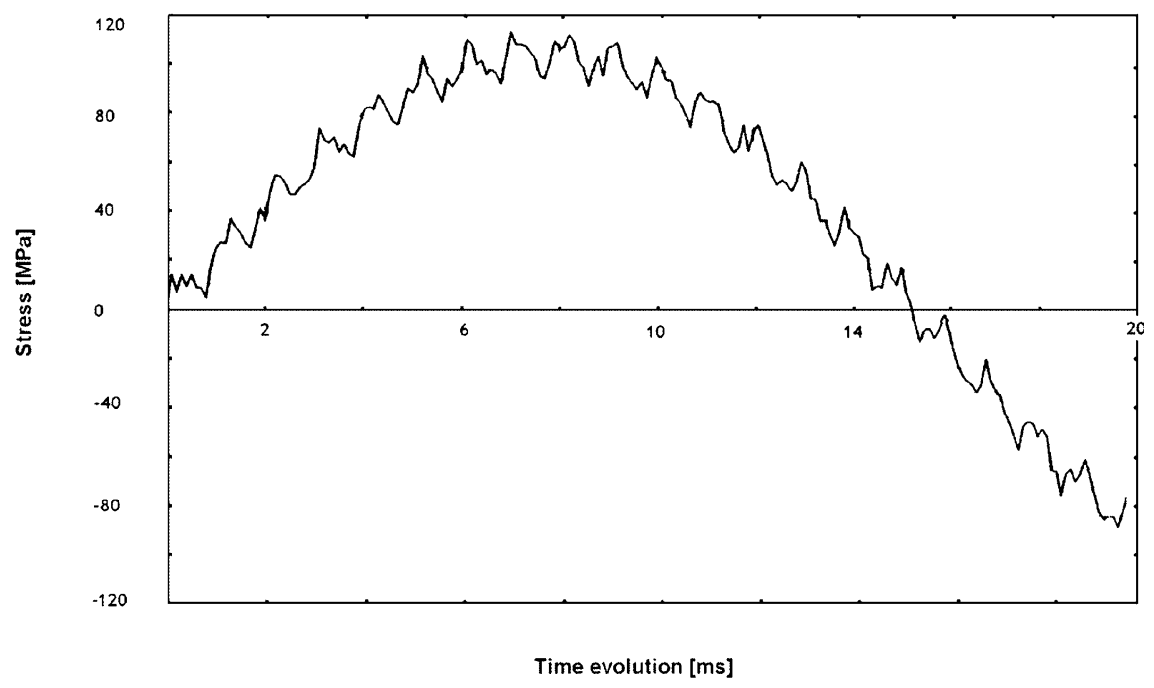

Fig. 16. Stress (MPa) at $(r=0, z=h / 2)$ vs. time (ms).

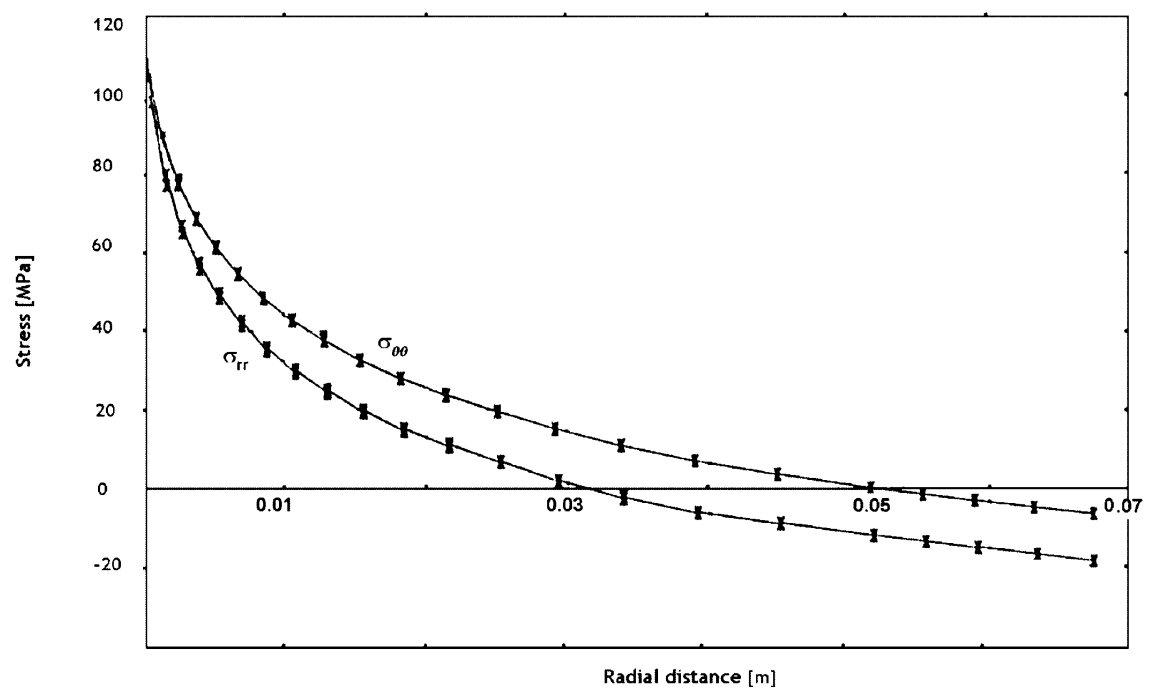

Fig. 17. Radial stress $\sigma_{r r}(\mathrm{MPa})$ and hoop stress $\sigma_{\theta \theta}(\mathrm{MPa})$ vs. radial distance $r(\mathrm{~m})$.

implies that if the value of the hoop stress is large enough, radial cracks may appear. However, according to the literature (see Bevan and Reed (1992) and Reed (1992)), the value of the fracture stress at such strain rates is around $145 \mathrm{MPa}$, which is larger than the maximum value of the hoop stress (115 MPa). Thus the plate is not damaged, which is confirmed by the experimental observation.

\section{Conclusion}

We have conducted a comprehensive impact signal analysis with four different methods: standard Fourier analysis, CWT, GT and Wigner-Ville transform. 
We were able to detect the different events characterizing discontinuities, and to read the frequency information contained in the signals. This yields a powerful tool for cleaning an experimental signal in a relevant way.

The frequency values revealed by the signal analysis correspond very well to the respiration frequencies computed by FE model analyses, when the inertia of the striker is taken into account. Also, the stresses obtained by numerical simulations are compatible with the experiment.

For a future work, the wavelet technique should be applied to a larger range of conditions and materials, and for the FE analyses, the following points should be investigated:

1. Take into account a viscoelastic behavior of the plate material (instead of the isotropic linear elasticity assumption (H3) of Appendix B).

2. Better modeling of the impact, e.g., contact with slip (and possibility of contact loss) between a rigid surface (the striker) and the plate.

3. Simulation of impact tests in which fracture has occurred. This will enable to validate the simple fracture criterion based on maximum principal tensile stress or try other criteria.

4. Perform geometrically nonlinear analyses, especially taking into account finite displacement effects (i.e., remove assumption (H2) of Appendix B).

\section{Acknowledgements}

Although the initial idea of this work goes back to discussions of AG with her colleague R. Tempels a few years ago, the decisive step was the suggestion of Y. Dauphin to use wavelets. AG thanks them both. We also thank B. Risse and S. Focant, who contributed some results and figures in their respective works Risse (1998) and Focant (1998).

\section{Appendix A. Ridges and skeleton of the continuous wavelet transform}

As explained in Section 2, plotting $|S(b, a)|$ as a function of scale $a$ and time $b$ often yields a noisy picture, difficult to interpret. For that reason, one generally uses the skeleton of the continuous wavelet transform (CWT) instead of its modulus. Roughly speaking, the skeleton is a collection of lines, called ridges, which are approximately lines of local maxima. For the purpose of the present analysis, it is useful to distinguish two different situations.

Assume first that the signal $s(t)$ consists of a singularity $\gamma_{\alpha}\left(t-t_{0}\right)$, of order $\alpha$, at time $t_{0}$, superimposed on a smooth, possibly harmonic, background and some stochastic noise, see Mallat and Hwang (1992). Here the singularity function $\gamma_{\alpha}$ is defined as follows:

$$
\gamma_{\alpha}\left(t-t_{0}\right)= \begin{cases}0, & t \leqslant t_{0} \\ \left(t-t_{0}\right)^{\alpha}, & t>t_{0}\end{cases}
$$

Thus we have

$$
\frac{\mathrm{d}^{\alpha+1}}{\mathrm{~d} t^{\alpha+1}} \gamma_{\alpha}\left(t-t_{0}\right)=\Gamma(\alpha+1) \delta\left(t-t_{0}\right)
$$

Let the wavelet be the $n$th derivative of a smooth positive function $\phi$, that is, $\psi(t)=\frac{\mathrm{d}^{n}}{\mathrm{~d} t^{n}} \phi(t)$, with $n \geqslant \alpha+1$. Then the CWT of $\gamma_{\alpha}$ with respect to $\psi$ reads: 


$$
S_{\gamma_{\alpha}}(b, a)=\Gamma(\alpha+1) a^{\alpha} \frac{\mathrm{d}^{n-\alpha-1}}{\mathrm{~d} t^{n-\alpha-1}} \phi\left(\frac{t_{0}-b}{a}\right)
$$

Assume now that the modulus of the $(n-\alpha-1)$ th derivative of $\phi(t)$ has $N$ maxima $\left\{\phi_{j}, j=1, \ldots, N\right\}$ at times $\left\{t_{j}, j=1, \ldots, N\right\}$. Then, for each $a$, the modulus $\left|S_{\gamma_{\alpha}}(b, a)\right|$ has $N$ maxima localized at times $\left\{b_{j}=a t_{j}+t_{0}, j=1, \ldots, N\right\}$, which converge toward $t_{0}$ as $a \rightarrow 0$. Furthermore, the maxima of $\left|S_{\gamma_{\alpha}}(b, a)\right|$ lie on $N$ lines, called (vertical) ridges $\left\{b=a t_{j}+t_{0}, j=1, \ldots, N\right\}$, which converge toward the singularity $t_{0}$ of the signal. Then one defines the $j$ th ridge function $r_{j}$ as the modulus of the CWT along the $j$ th ridge:

$$
r_{j}(a)=\left|S_{\gamma_{\alpha}}\left(b=a t_{j}+t_{0}, a\right)\right|=\Gamma(\alpha+1) a^{\alpha} \phi_{j},
$$

so that

$$
\ln r_{j}(a) \sim \alpha \ln a+\ln \phi_{j} .
$$

Thus, in a $\log -\log$ plot, the ridge function $r_{j}$ is a straight line with slope equal to the order $\alpha$ of the singularity (see Alexandrescu et al. (1996) for a pedagogical discussion of this technique). In the present paper, the signal describing a rupture of the sample will have a jump singularity $(\alpha=0)$ (that is, $\gamma_{\alpha}$ is a Heaviside function), hence a first derivative of a Gaussian suffices to detect it. However, the second derivative (the Mexican hat wavelet), will do also. In addition, in the case of a rebound, it detects the precise instants of loading and unloading (beginning and end of contact between the striker and the sample), which correspond to discontinuities in the derivative of the signal (Fig. 5). Thus, it is useful to use them both.

For the second typical situation we assume that the signal is well approximated by a superposition of spectral lines:

$$
s(t)=\sum_{j=1}^{N} s_{j}(t), \quad s_{j}(t)=A_{j}(t) \mathrm{e}^{\mathrm{i} \omega_{j} t}
$$

where the amplitude $A_{j}(t)$ varies slowly. By linearity, the WT of this signal is a sum of terms, $S(b, a)=\sum_{j} S_{j}(b, a)$, where

$$
\begin{aligned}
S_{j}(b, a) & =\sqrt{a} \int_{-\infty}^{+\infty} \widehat{\psi}(a \omega) \widehat{A}_{j}\left(\omega-\omega_{j}\right) \mathrm{e}^{\mathrm{i} \omega b} \mathrm{~d} \omega, \\
& =\sqrt{a} \mathrm{e}^{\mathrm{i} \omega_{j} b} \int_{-\infty}^{+\infty} \widehat{\psi}\left(a\left(\omega+\omega_{j}\right)\right) \widehat{A}_{j}(\omega) \mathrm{e}^{\mathrm{i} \omega b} \mathrm{~d} \omega .
\end{aligned}
$$

Using the Taylor expansion of $\widehat{\psi}$ around the pulsation $\omega_{j}$,

$$
\widehat{\psi}\left(a\left(\omega+\omega_{j}\right)\right)=\widehat{\psi}\left(a \omega_{j}\right)+\sum_{k} \frac{(a \omega)^{k}}{k !} \frac{\mathrm{d}^{k} \widehat{\psi}}{\mathrm{d} \omega^{k}}\left(a \omega_{j}\right),
$$

we obtain the following expansion for $S_{j}$

$$
S_{j}(b, a)=\widehat{\psi}\left(a \omega_{j}\right) s_{j}(b)+\mathrm{e}^{\mathrm{i} \omega_{j} b} \sum_{k \geqslant 1} \frac{(-\mathrm{i} a)^{k}}{k !} \frac{\mathrm{d}^{k} \widehat{\psi}}{\mathrm{d} \omega^{k}}\left(a \omega_{j}\right) \frac{\mathrm{d}^{k} A_{j}}{\mathrm{~d} b^{k}}(b) .
$$

So one has

$$
S_{j}(b, a) \simeq \widehat{\psi}\left(a \omega_{j}\right) s_{j}(b),
$$


up to first order, and

$$
S(b, a) \simeq \sum_{j=1}^{N} \widehat{\psi}\left(a \omega_{j}\right) s_{j}(b) .
$$

Assume that the wavelet $\widehat{\psi}(\omega)$ has a unique maximum in frequency space at $\omega=\omega_{0}$, like the Morlet wavelet. Then, if the values of the frequencies $\omega_{j}$ are sufficiently far away from each other, the factor $\widehat{\psi}\left(a \omega_{j}\right)$ allows to treat each spectral line independently. In this case, the contribution of the $j$ th spectral line to $S(b, a)$ is localized on the scale $a_{j}=\omega_{0} / \omega_{j}$ and, along the line of maxima $a=a_{j}$, called the $j$ th (horizontal) ridge, the CWT is approximately proportional to the $j$ th spectral line:

$$
\frac{S\left(b, a_{j}\right)}{\widehat{\psi}\left(\omega_{0}\right)} \simeq s_{j}(b) .
$$

The set of all the ridges is again called the skeleton of the CWT. Thus the restriction of the WT $S(b, a)$ to its skeleton contains the whole information.

The analysis extends to the more general case where the spectral lines have the form

$$
s_{j}(t)=A_{k}(t) \mathrm{e}^{\mathrm{i} \phi_{j}(t)},
$$

where the amplitude $A_{j}(t)$ varies slowly with respect to the phase $\phi_{j}(t)$ (such signals are called asymptotic). In this case, each term $S_{j}$ in the CWT (2.2) in the time domain is a rapidly oscillating integral, the essential contribution to which is given by the stationary points of the phase of the integrand. These points are the solutions $t_{s}(a)$ of the equation

$$
\frac{\mathrm{d} \phi_{j}}{\mathrm{~d} t}\left(t_{s}\right)=\frac{\omega_{0}}{a} .
$$

Then the corresponding ridge of the WT is defined as the set of points $(a, b)$ for which $t_{s}(a)=b$. These constitute a curve in the $(a, b)$-half-plane, which essentially reduces to a line of local maxima. A detailed analysis (see Delprat et al. (1992)) shows again that, on this curve, the WT $S(b, a)$ coincides, up to a small correction, with the component $s_{j}(b)$ of the signal.

\section{Appendix B. Kirchhoff-Love and Reissner-Mindlin plate theories}

The KL theory for bending of thin plates is based on the following assumptions:

(H1) The plate has a uniform thickness $h$; its middle plane is $(z=0)$.

(H2) The strains, displacements and rotations are small.

(H3) The material behavior obeys isotropic linear elasticity.

(H4) Material segments, which are initially normal to the middle plane remain straight and normal to the deformed middle surface. This implies that the transverse shear strains are nil $\left(\epsilon_{x z}=\epsilon_{y z}=0\right)$. Thus, the transverse shear stresses also vanish $\left(\sigma_{x z}=\sigma_{y z}=0\right)$.

(H5) The plate is inextensible in the normal direction. Consequently, the out-of-plane strain is zero $\left(\epsilon_{z z}=0\right)$ and the transverse displacement $w$ is independent of $z$, i.e. $w=w(x, y)$.

(H6) The normal stress is negligible in front of the in-plane stresses $\left(\sigma_{z z} \approx 0\right)$. 
Given these assumptions, it can be shown (see, for instance, Timoshenko and Woinowsky-Krieger (1959)) that the problem of bending of a thin plate is reduced to solving the following partial differential equation for transverse displacement $w$ (under appropriate boundary and initial conditions):

$$
D \Delta \Delta w+m \ddot{w}=p,
$$

where $D(\mathrm{Nm})$ is the flexural rigidity, $m\left(\mathrm{~kg} / \mathrm{m}^{2}\right)$ the mass per unit area and $p\left(\mathrm{~N} / \mathrm{m}^{2}\right)$ the pressure:

$$
D \equiv \frac{E h^{3}}{12\left(1-v^{2}\right)}, m \equiv \int_{-h / 2}^{h / 2} \rho \mathrm{d} z
$$

with $E\left(\mathrm{~N} / \mathrm{m}^{2}\right)$ being Young's modulus, $v$ Poisson's ratio and $\rho\left(\mathrm{kg} / \mathrm{m}^{3}\right)$ the mass density.

The RM plate theory has the same assumptions as those of KL except assumption (H4) which is replaced with the following:

"(H4-RM) Material segments, which are initially normal to the middle plane remain straight, but not necessarily normal to the deformed middle surface."

This implies that the transverse shear strains $\left(\epsilon_{x z}\right.$ and $\left.\epsilon_{y z}\right)$ do not vanish, and consequently neither do the transverse shear stresses $\left(\sigma_{x z}\right.$ and $\left.\sigma_{y z}\right)$.

RM theory is particularly well suited for thick plates and/or when transverse shearing cannot be neglected.

\section{References}

ABAQUS finite element program, 1998, Hibbitt, Karlsson \& Sorensen, Inc., Pawtucket, RI.

Alexandrescu, M., Gibert, D., Hulot, G., Le Mouel, J.L., Saracco, G., 1996. Worldwide wavelet analysis of geomagnetic jerks. J. Geophys. Res. B 101, 21975-21994.

Antoine, J.-P., Coron, A., Dereppe, J.-M., 2000. Water peak suppression: time-frequency vs. time-scale approach. J. Magnetic Reson A $144,189-194$.

Bevan, L., Reed, P.E., 1992. Impact failure of poly(methyl methacrylate). J. Appl. Poly. Sci. 46, 2131-2137.

Daubechies, I., 1992. Ten Lectures on Wavelets. SIAM, Philadelphia, PA.

Delprat, N., Escudié, B., Guillemain, Ph., Kronland-Martinet, R., Tchamitchian, Ph., Torrésani, B., 1992. Asymptotic wavelet and gabor analysis: extraction of instantaneous frequencies. IEEE Trans. Inform. Theory 38, 644-664.

Feichtinger, H.G., Strohmer, T. (Eds.), 1998. Gabor Analysis and Algorithms - Theory and Applications. Birkhäuser, Boston-BaselBerlin.

Flandrin, P., 1993. Temps-fréquence. Hermès, Paris.

Focant, S., 1998. L'analyse temps-fréquence de Wigner-Ville permet une étude détaillée des signaux de fracture. Thèse annexe, Univ. Cath. Louvain, Louvain-la-Neuve.

Hughes, T.J.R., 1987. The Finite Element Method. Prentice-Hall, Englewood Cliffs, NJ.

Mallat, S., Hwang, W.L., 1992. Singularity detection and processing with wavelets. IEEE Trans. Inform. Theory 38, $617-643$.

Mallat, S.G., 1999. A Wavelet Tour of Signal Processing, second edn.. Academic Press, San Diego, CA.

Maurer, G., Breuer, H., 1995. Instrumented impact test: influence of shape and material of the striking fin on the force-time trace. In: Williams, J.G., Pavan, A. (Eds.), Impact and Dynamic Fracture of Polymers and Composites. ESIS 19, Mechanical Engineering Publications, London, pp. 93-102.

Mecklenbräuker, W., Hlawatsch, F. (Eds.), 1997. The Wigner Distribution. Elsevier, Amsterdam.

Meyer, Y. (Ed.), 1991. Wavelets and Applications. Proc. Marseille 1989, Springer, Berlin.

Meyer, Y., Roques, S. (Eds.), 1993. Progress in Wavelet Analysis and Applications. Proc. Toulouse 1992, Editions Frontières, Gif-surYvette.

Meyer, Y., 1992. Les Ondelettes, Algorithmes et Applications. Armand Colin, Paris (Transl.; Wavelets, Algorithms and Applications. SIAM, Philadelphia, PA, 1993).

Reed, P.E., 1992. Falling weight impact testing and design. Plast. Rub. Comp. Appl. 17, 157-163. 
Risse, B., 1998. Analyse de poids tombant instrumenté par les ondelettes et les éléments finis. Mémoire MECA, Univ. Cath. Louvain, Louvain-la-Neuve.

SAMCEF finite element program, 1997, SAMTECH, S.A., Liège, Belgium.

Savadori, A., 1985. Impact testing of plastics: present knowledge. Polym. Test. 5, 209-241.

Timoshenko, S., Woinowsky-Krieger, S., 1959. Theory of Plates and Shells. McGraw-Hill, New York, NY.

Torrésani, B., 1995. Analyse continue par ondelettes. InterEditions, CNRS Editions, Paris.

Van Vyve, E., 1997. Analyse d'un test de choc sur des matériaux polymères par la méthode des ondelettes et de l'élasticité. Mémoire MECA, Univ. Cath. Louvain, Louvain-la-Neuve. 